\title{
Dificultades en la escritura académica de estudiantes de maestría
}

\author{
Academic Writing Difficulties of Master's Students \\ Dificuldades na escrita acadêmica de estudantes do mestrado
}

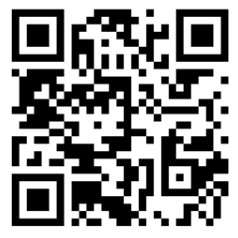

\author{
Marisol Rey-Castillo \\ Tecnológico de Monterrey \\ Monterrey, México \\ marisol.rey@teachers.org \\ iD http://orcid.org/0000-0003-4277-9545 \\ Marcela Georgina Gómez-Zermeño \\ Tecnológico de Monterrey \\ Monterrey, México \\ marcela.gomez@tec.mx \\ iD http://orcid.org/0000-0002-5427-2891
}

Recibido • Received • Recebido: 07 / 06 / 2019

Corregido • Revised • Revisado: 12 / $01 / 2021$

Aceptado • Accepted • Aprovado: $16 / 02 / 2021$

\begin{abstract}
Resumen: La alfabetización académica es el proceso que permite el desarrollo de competencias necesarias para la comprensión y producción de textos en el ámbito de la educación superior. Esta se construye a lo largo de la formación profesional y continúa incluso en el postgrado, por lo que siempre existirán áreas de oportunidad y mejora de acuerdo con las necesidades de comunicación académica y científica. El objetivo de este trabajo fue identificar dificultades en la escritura académica de estudiantes de dos programas de maestría en línea de una universidad en el noreste de México. A través del análisis cualitativo de doce muestras escritas del estudiantado y entrevistas a cinco docentes, se pudo identificar que aspectos como la documentación, la articulación de la propia voz con las fuentes, la citación y algunos elementos de redacción son susceptibles de ser mejorados.
\end{abstract}

Palabras claves: Escritura académica; alfabetización académica; habilidades de escritura; competencia comunicativa escrita; postgrado.

Abstract: Academic literacy is the process that allows the development of necessary skills for the comprehension and production of texts in the field of higher education. This literacy is built across undergraduate studies and continues even in postgraduate education, so there will always be areas of opportunity and improvement according to academic and scientific communication needs. This work aimed to identify difficulties in the academic writing of students from two online master's programs from a university in northeastern Mexico. Through the qualitative analysis of twelve students'texts and interviews with five teachers, it was possible to identify aspects that can be improved, such as documentation, the articulation of one's own voice with the sources, the citation, and some drafting elements.

Keywords: Academic writing; academic literacy; writing skills; written communicative competence; graduate education. 
http://doi.org/10.15359/ree.25-2.4

http://www.una.ac.cr/educare

educare@una.ac.cr

\begin{abstract}
Resumo: A alfabetização acadêmica é o processo que permite o desenvolvimento de habilidades necessárias para a compreensão e produção de textos no ensino superior. Esta é construída em toda a graduação e continua até mesmo na pós-graduação, portanto sempre haverá áreas de oportunidade e aperfeiçoamento, de acordo com as necessidades da comunicação acadêmica e científica. Este trabalho teve como objetivo identificar dificuldades na escrita acadêmica de estudantes de dois programas de mestrado on-line em uma universidade no nordeste do México. Através da análise qualitativa de doze amostras escritas dos estudantes e entrevistas com cinco de seus professores, foi possível identificar que aspectos como documentação, articulação da própria voz com as fontes, a citação e alguns elementos de redação poderiam ser melhorados.
\end{abstract}

Palavras-chave: Redação acadêmica; alfabetização acadêmica; habilidades de escrita; competência comunicativa escrita, pós-graduação.

\title{
Introducción
}

\section{Alfabetización académica en postgrado}

La competencia comunicativa escrita hace parte del grupo de competencias transversales que deben desarrollar y poner en práctica profesionales integrales (Argudín y Luna, 2005; Carlino, 2005). Esta implica el dominio y uso de conceptos y estrategias para comunicarse por escrito en un contexto determinado. En la educación superior, esto se denomina alfabetización académica (Carlino, 2003, 2013; Radloff y de la Harpe, 2000) que se refiere al grupo de conocimientos, nociones y estrategias que le permiten al estudiantado producir textos académicos en el contexto de la educación superior, articulando los saberes de su disciplina con la competencia comunicativa escrita. Esto permite, no solo el buen desempeño textual, sino la posibilidad de pertenecer a una comunidad académica y profesional (Flores Aguilar, 2014).

Esta competencia también sirve de anclaje para el desarrollo de otras habilidades cognitivas como la organización, la estructuración de ideas, la argumentación y el pensamiento crítico, entre otros (Argudín y Luna, 2005; Carlino, 2005). De allí la importancia de que el desarrollo de esta se convierta en un elemento fundamental en la formación universitaria. En el contexto del postgrado, esta se centra en la escritura de textos propios de este nivel como las tesis, las monografías, las ponencias y los artículos de investigación, entre otros (Aguilar González y Fregoso Peralta, 2016; Bandenhorst y Guerin, 2016; Stillman-Webb, 2016).

La escritura no puede concebirse como una acción, sino como un proceso en el que quiene escribe pone en juego diversas estrategias cognitivas que requieren de variadas habilidades (Hayes, 1996), no obstante, esta contiene elementos evidenciables en el texto susceptibles de ser analizadas por separado. Esto permite ser conscientes de los distintos aspectos que la envuelven y, por consiguiente, mejorarlos. Siguiendo a Aguilar González y Fregoso Peralta (2016) y a Cassany $(2004,2006)$, se presentan, en la Figura 1, los aspectos inherentes al texto. 
http://doi.org/10.15359/ree.25-2.4

Figura 1: Aspectos inherentes al texto

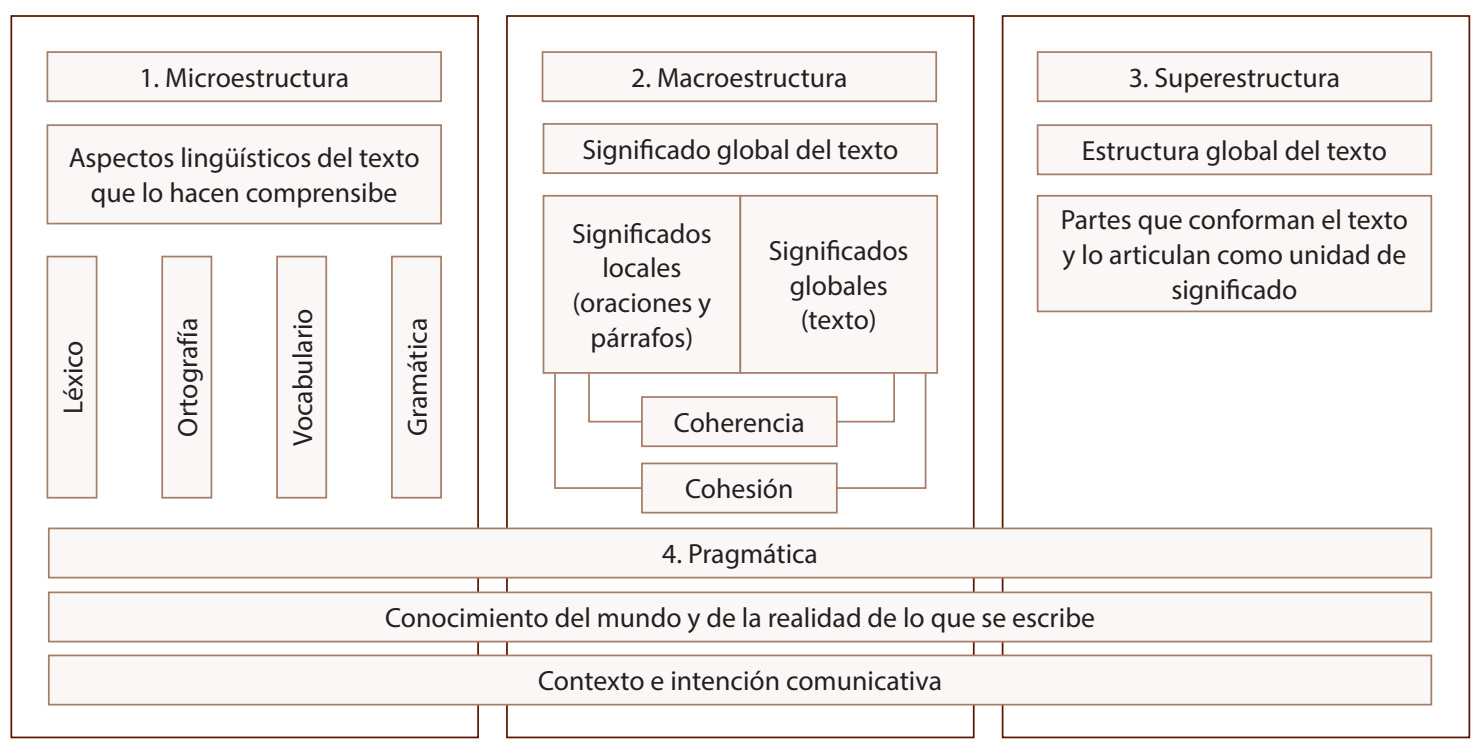

Nota: Elaboración propia.

El texto académico tendrá sentido solo si los significados que se encuentran en él son producto de una actividad en la que se comprenda y se tenga una postura ante un conocimiento. De tal manera que el acto de escritura nunca viene antes de un conocimiento derivado de una búsqueda documental que le permita apropiarse de las concepciones que va a expresar en el escrito. La documentación implica una búsqueda, selección y lectura de información relevante que permita, al escritor o escritora, configurar unas nociones propias sobre el tema planteado (Argudín y Luna, 2005; Carlino, 2005, 2013).

Asimismo, un texto debe expresar ideas propias que plasman una postura crítica ante el conocimiento que presenta. En este sentido, es importante que el autor o autora de un texto sepa articular su propia voz (sus ideas, concepciones y opiniones) a los conocimientos de los textos han servido para el aprendizaje y la construcción de su saber (Carlino, 2003, 2013).

\section{Dificultades en la producción escrita}

Carlino (2004) identifica una serie de dificultades comunes en el estudiantado universitario. Entre estas: 1) no tener en cuenta el contexto de producción y la articulación de las fuentes con su propia voz; 2) prosa con una estructura de temas idéntica a la de sus fuentes de información; 3) poca adecuación del tema, no solo al propósito de su escrito, sino también a su potencial lector; 4) escasa profundidad en la revisión de sus propios escritos y centrada en aspectos de forma, pero no de contenido; 5) poca planeación de la escritura y de la manera en la que el estudiantado se acerca al proceso mismo. 
http://doi.org/10.15359/ree.25-2.4

http://www.una.ac.cr/educare

educare@una.ac.cr

En el nivel de postgrado, existen, además de los ya mencionados, otros factores problemáticos. Bandenhorst y Guerin (2016) mencionan que, si bien la competencia comunicativa escrita debe desarrollarse de manera transversal en el nivel de postgrado, al igual que se hace en la formación profesional, existe poca sistematicidad en los hábitos, las rutinas y las prácticas, por la misma naturaleza de este nivel educativo, lo que conduce a una serie de dificultades en la construcción del texto escrito, tanto en la forma, como en el contenido. Por otra parte, Stillman-Webb (2016) asegura que, en este nivel académico, a pesar de existir propósitos formativos distintos a los del pregrado, es necesario generar un acompañamiento similar a través de diversos mecanismos como la tutoría, los talleres, los cursos y otros que permitan el acompañamiento al estudiantado.

\section{Estudios previos en nivel de postgrado}

A partir de la necesidad de desarrollar habilidades en la escritura académica de estudiantes en la universidad, se generó el interés por integrar, a la formación profesional, distintas estrategias para acompañar al estudiantado en el proceso de escritura por parte de las instituciones de educación superior. Así nacen distintos programas materializados en talleres, centros de escritura, creación de cursos anclados al currículo o como asignatura electiva, manuales y cartillas, entre otros. De igual manera se actualiza al personal docente para proporcionarle herramientas que puedan implementar en sus clases y que potencien el desarrollo de la competencia comunicativa escrita en sus estudiantes (Carlino, 2004, 2013).

En el nivel de postgrado, se han realizado diversos estudios en torno a las características de la escritura del estudiantado y los programas de intervención planteados para el mejoramiento de la competencia escrita. Algunos de ellos se han enfocado en el análisis de documentos escritos por estudiantes, ya sea tesis de maestría y ponencias de estudiantes en este nivel (Aguilar González y Fregoso Peralta, 2016; Arnoux et al., 2004; Martín Torres, 2012; Padilla, 2016), o artículos y ensayos realizados por estudiantes de doctorado (Bosio, 2018; Padilla, 2016) con el fin de identificar áreas de oportunidad y mejoramiento para realizar intervenciones en el aula a partir de talleres y asesoría de docentes o personal experto, para fortalecer las habilidades de escritura. De igual manera, otros estudios se han enfocado en el desarrollo de programas, planes, talleres y tutorías o asesorías (Pereira y di Stefano, 2007; Rodríguez Hernández y García Valero, 2015; Salinas Padilla et al., 2009; Stillman-Webb, 2016) en cursos de maestría en los que, a través de la intervención de los equipos investigadores y expertos, se han identificado una serie de dificultades y retos en el proceso de escritura del alumnado.

Estos estudios, entre otros no nombrados aquí, permiten vislumbrar no solo que el desarrollo de la competencia comunicativa escrita no se culmina en el nivel profesional, sino que es susceptible de ser perfeccionada a lo largo del postgrado, razón por la cual se convierte en un foco de estudio.

4 
De esta manera, surgió la necesidad de preguntar: ¿cuáles son las dificultades en la competencia comunicativa escrita para la elaboración de textos académicos de estudiantes de dos programas de maestrías en una universidad mexicana? La respuesta a esta pregunta puede llegar a constituirse como un diagnóstico para emprender acciones que permitan mejorar los aspectos encontrados y con ello contribuir al perfeccionamiento de dicha competencia.

El propósito de este texto es detallar las dificultades de la competencia comunicativa escrita en el estudiantado de dos programas de maestría en el área de educación, en una universidad mexicana, evidenciadas a través de los escritos de tales estudiantes y de lo identificado por sus docentes.

\section{Materiales y métodos}

Dado que el objetivo del presente estudio era identificar las deficiencias en la competencia comunicativa escrita para la elaboración de textos académicos del estudiantado de maestría en un lugar concreto, se optó por un enfoque cualitativo, pues este permite comprender la realidad en el contexto elegido a partir de un acercamiento al objeto de estudio para reconocer y describir dicha realidad (Denzin y Lincoln, 2011; Maxwell, 2013).

Como escenario del estudio se eligió una institución de educación superior del noreste de México, específicamente una Facultad de Humanidades, de la cual se seleccionaron dos programas de maestría en línea, relacionados con las ciencias de la educación. Aunque la universidad que ofrece los programas académicos se encuentra en México, gracias a diversos convenios y a su misma presencia en otros países, recibe estudiantes de Centroamérica, Sudamérica y España.

Se optó por el muestreo con propósito (Maxwell, 2013), el cual permite seleccionar una población específica, en este caso, la escritura académica en el postgrado. Para este caso, se analizaron doce muestras escritas por estudiantes, cada una con una extensión promedio de 2000 palabras. Además, se entrevistaron cinco docentes con función tutorial de estas personas autoras de los textos: cuatro están a cargo de asignaturas relacionadas con la investigación que llevará el estudiantado a la escritura de su trabajo de fin de maestría; el otro docente es titular de una cátedra en la que existe abundante producción escrita.

Esta selección de docentes se justifica dado que se encuentran en contacto permanente con la producción escrita del estudiantado y pueden identificar los rasgos característicos de los textos, los que pueden evidenciarse a través de las muestras escritas. Se decidió no entrevistar estudiantes, pues no siempre son conscientes de sus áreas de mejoramiento, o podrían sentir que se les critica, si admiten tener dificultades en sus procesos de escritura y, por ello, no proporcionar información verdadera. 
http://doi.org/10.15359/ree.25-2.4

http://www.una.ac.cr/educare

educare@una.ac.cr

En consonancia con las fuentes de información, se utilizaron dos instrumentos para recolección de datos: el análisis documental y la entrevista. El análisis documental (García Gutiérrez, 2002; Ruiz Pérez, 1992) permite el acercamiento a un documento para identificar elementos susceptibles de ser definidos o clasificados y utilizados posteriormente en un análisis. En este caso, se usó para el estudio de las muestras de escritura, a partir del establecimiento de criterios relacionados con la forma escrita en cuatro dominios: documentación en distintas fuentes, forma del escrito (ortografía, gramática y vocabulario formal y del campo académico), redacción de oraciones y párrafos, y articulación de sus propias voces con las fuentes.

Por otro lado, se diseñó una entrevista semiestructurada para docentes, con nueve preguntas abiertas que permitieron identificar puntos específicos relacionados con los mismos dominios del análisis de las muestras. Al ser semiestructurada, se posibilitó la introducción de nuevas preguntas o la profundización de algún aspecto a partir de la interacción con los sujetos entrevistados (Valenzuela González y Flores Fahara, 2012; Vela, 2001). Las entrevistas fueron grabadas en audio y transcritas usando las convenciones de Sacks et al. (1974).

Por lo que respecta al proceso de acercamiento a la población y la recolección de los datos, se siguieron una serie de pasos que se muestran en la Figura 2.

Figura 2: Procedimiento para recolección de datos

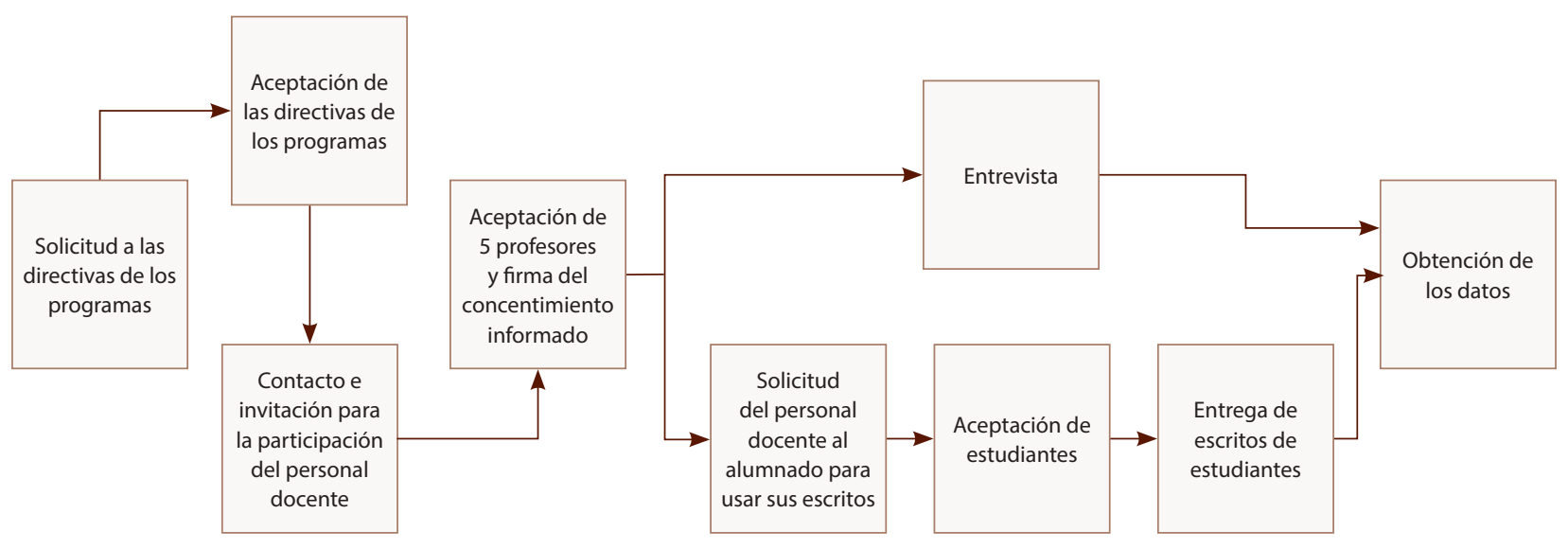

Nota: Elaboración propia.

El análisis de datos se apoyó en el software ATLAS.ti versión 7.5.4. Se creó una unidad hermenéutica en la cual se adjuntaron las transcripciones de las cinco entrevistas y las doce muestras de escritura. A partir del estudio de los datos, se crearon veintidós categorías que fueron agrupadas en seis familias. Estas se señalaron en el texto generando 236 citas extraídas de los documentos y las entrevistas. La validez de los datos se controló a partir de la triangulación entre lo hallado en las entrevistas y su correlato en las muestras de escritura. 


\section{Resultados}

\section{La población y la escritura académica en los programas}

La población de estudiantes en los programas de maestría es heterogénea. Las cinco personas docentes coinciden en que se trata de una población variada en muchos sentidos: áreas de conocimiento, procedencia geográfica, intereses y motivaciones para cursar la maestría y experiencias previas en torno a la escritura. Esto es un elemento que influye en el desempeño de las actividades escritas, asunto que se evidencia en la producción de textos como en el resto de tareas académicas, y que deriva en distintas características evidenciables en el texto.

¿Quiénes son los que (0.2) este, he batallado a lo mejor más? Con las personas que no han tenido como ese contacto de hacer este un trabajo de investigación, inclusive una tesis. (0.2) A veces es como si fueras a dar un taller de escritura nivel 1, porque hay gente que está en nivel 8, y hay gente que tiene a lo mejor nivel 8, por el grado, por lo que ha trabajado y por lo que ha vivido. (Profesor \#1)

Las distintas formas en la escritura señaladas por el grupo de docentes son evidentes en los textos de la muestra, pues mientras unos textos están cercanos a la forma estandarizada del registro escrito (Muestra \#6), otros dejan ver dificultades desde la ortografía, el uso de vocabulario y términos, la construcción de oraciones y párrafos y la articulación de estos (Muestra \#3). Esta característica es común en este tipo de nivel educativo (Aguilar González y Fregoso Peralta, 2016; Padilla, 2016; Stillman-Webb, 2016).

De manera similar, en la formación académica en postgrado, la escritura se visualiza como una competencia transversal a otras competencias relacionadas con la construcción de conocimientos en este nivel y con la investigación. El profesorado entrevistado mencionó la escritura y todos los elementos que la componen como un aspecto fundamental, no solamente en su espacio académico, sino como algo relevante en el desarrollo formativo del estudiantado:

Se planea que el curso y las actividades abonen en el desarrollo de esas competencias disciplinares y transversales, y parte del cómo está estructurado escribiendo, redactando, investigando. (Profesor \#2)

Este pensamiento de la escritura como competencia trasversal relevante en el desarrollo de la formación postgradual ya había sido notado por estudios previos (Arnoux et al., 2004; Bandenhorst y Guerin, 2016; Martín Torres, 2012).

Asimismo, el profesorado señala distintos recursos que sus estudiantes utilizan en este contexto como herramientas fundamentales que apoyan al desarrollo de esa competencia. Dos mencionan el uso de fichas bibliográficas como apoyo en el proceso de documentación. 
http://doi.org/10.15359/ree.25-2.4

http://www.una.ac.cr/educare

educare@una.ac.cr

También, dos de sus docentes argumentan la modelación como una forma en la que el estudiantado puede mejorar esta competencia escrita, asunto que ya había sido detallado en otros estudios (Bosio, 2018; Martín Torres, 2012).

Las fichas bibliográficas que ellos deben hacer también son parte necesaria que les ayuda mucho. (Profesor \#4)

Pero ya después de que lo hago yo, que ya hago el modelaje, por medio de mi escrito, entonces les digo "ahora ustedes". (Profesor \#1)

Como caso particular, los dos programas de maestría cuentan con un manual de escritura publicado por la institución y diseñado para establecer lineamientos que permitan, por un lado, proporcionar guía al estudiantado para una escritura correcta, por otro lado, unificar criterios en los distintos tipos de documentos que se trabajan en estos dos programas.

Ahí, tenemos que hacer mucho trabajo de promoción del manual. Algunos alumnos lo utilizan, desde el principio son muy aplicados lo toman por su cuenta, y en algunos otros alumnos, sí tenemos que estar insistiendo en que revisen el manual. (Profesor \#3)

Adicionalmente, un profesor informa otros recursos con los que cuenta la institución de educación superior: una página web con información adicional y el apoyo que proporcionan las capacitaciones de la biblioteca en torno a los temas de documentación, citación y referenciación.

Lo anterior señala que no solamente existen mecanismos al interior de los cursos para apoyar la escritura del estudiantado, sino también un interés institucional en el desarrollo de estrategias que apoyen y unifiquen criterios de escritura. El uso de manuales y otros recursos, como incluso talleres, grupos de trabajo y otras herramientas aledañas a los cursos ya había sido evidenciado en trabajos anteriores sobre escritura en el postgrado (Bosio, 2018; Martín Torres, 2012; Pereira y di Stefano, 2007; Stillman-Webb, 2016).

De igual manera, el grupo docente señala que existe una progresión en la escritura y que la competencia va mejorando paulatinamente:

También se aprende así. Y ya algunos, captan la idea perfectamente y ya no lo hacen, lo cuidan. (Profesor \#3)

Al principio les cuesta un poco, pero poco a poco van mejorando con lo que ellos mismos van trabajando. (Profesor \#5)

La progresión en la escritura académica también ha sido señalada en estudios anteriores en los que se sustenta la incorporación de distintas estrategias a partir de la retroalimentación recibida (Martín Torres, 2012; Stillman-Webb, 2016). 
http://doi.org/10.15359/ree.25-2.4

\section{Documentación como base de la escritura académica}

En el contexto académico, la escritura está dirigida a la comunicación del conocimiento en las distintas disciplinas, por consiguiente, esta parte de una base documental en la cual el estudiantado acopia una serie de conocimientos, nociones o ideas, que se convertirán en parte del contenido. En ese sentido, la documentación se convierte en uno de los elementos principales en el proceso de escritura. Para el caso particular, todo el grupo docente manifestó que existen lecturas básicas en el contexto de la asignatura:

Yo les comparto una lista, dependiendo de cada tema que a ellos les interese este (0.5) trabajar en su proyecto de investigación (0.5) y entonces de ahí parto yo (Profesor \#4).

Adicionalmente, aunque coinciden en que esta documentación debe verse complementada a partir de la búsqueda independiente que debe realizar cada estudiante a partir de sus inquietudes intelectuales, una parte afirma que en la fase de documentación propia existen algunas deficiencias, como no lograr exhaustividad, no atender a distintos tipos de fuentes o acudir a fuentes desactualizadas:

$Y$ en ocasiones buscan los artículos cuando se les piden actualizados, a veces fallan en eso. Entonces la pregunta es si ellos toman en cuenta eso, sí, algunos alumnos son muy aplicados y otros no. Otros no son aplicados (Profesor \#3).

Por otro lado, dos indican dificultades del estudiantado para seleccionar información pertinente de acuerdo con la temática de su investigación y el propósito comunicativo de su escrito:

Fallan en esa búsqueda de actualización, como que no es importante para ellos en las fechas, en las que están escritos los artículos en los que se han basado (0.5). Entonces, eso es para las cuestiones de resultados de investigación empírica que les ayuden a entender, que hay otros estudios, que hay antecedentes, que hay otras personas que han investigado lo mismo, o muy relacionado con lo que ellos están investigando (0.5). (Profesor \#3)

En todas las muestras de textos de estudiantes se evidencia que se siguió una documentación previa para la construcción del texto propio. Esto se manifiesta, no solo a través de las citas y referencias presentes, sino también del dominio del contenido en cada texto.

Las características y dificultades encontradas coinciden con experiencias previas en las que la selección de la información y el acopio de la documentación se han identificado como asuntos problemáticos para el estudiantado (Carlino, 2004; Rodríguez Hernández y García Valero, 2015). 
http://doi.org/10.15359/ree.25-2.4

http://www.una.ac.cr/educare

educare@una.ac.cr

\section{Forma del escrito}

Uno de los aspectos que la tecnología ha ayudado a solucionar, es el de la ortografía literal y acentual, pues como el mismo profesorado lo refiere, los procesadores de texto indican el error para solucionarlo. Los pocos errores que pueden existir corresponden más a fallas de digitación, que no es en realidad un error de ortografía por desconocimiento de la norma.

No lo hacen pienso yo, por falta de conocimiento de reglas ortográficas, que pasen y que escriban (0.3) una palabra, pensando que se escriba así, no es tanto eso, es más bien un error de dedo, podría decirse. (Profesor \#2)

Esto se observa en las muestras del estudiantado, pues en algunos casos existen leves errores de digitación, y en otros casos duda en cuanto al uso de mayúscula inicial en algunas palabras:

El programa busca formar Auxiliares en Enfermería con competencias laborales propuestas por el Ministerio de Educación Nacional. (Muestra \#5)

En lo que se identifica un área por mejorar es en el uso de signos de puntuación:

En lo que fallan es en los signos de puntuación, que es donde se puede ver mayor (0.4) cantidad o frecuencia de fallas, puesto que, que el Word te va corrigiendo la ortografía. (Profesor \#3)

Esto se ve corroborado en algunas muestras de escritura, en las que varios segmentos presentan errores: no hay presencia de un signo de puntuación que debería estar, o se utiliza un signo de puntuación inadecuado:

Está dando sus primeros resultados, al preparar jóvenes con competencias de: Se expresa y comunica, Piensa crítica y reflexivamente. (Muestra \#12)

Con respecto al tercer aspecto: El rol del docente y el estudiante en el proceso de enseñanza aprendizaje, se identifica que constantemente se están desarrollando diferentes herramientas, recursos, programas, etc. (Muestra \#2)

Esto coincide con algunos trabajos previos en los que se logró caracterizar las deficiencias en signos de puntuación como uno de los puntos necesarios a fortalecer en la escritura académica del estudiantado (Aguilar González y Fregoso Peralta, 2016; Salinas Padilla et al., 2009).

Por lo que se refiere a la redacción, el profesorado señala que sus estudiantes usan la primera persona del singular, incluso cuando se les ha recomendado de manera expresa que deben utilizar la segunda de pasiva: 
http://doi.org/10.15359/ree.25-2.4

Lo que batallan es en el tiempo. Utilizan (0.2) lo que más pueden utilizar es la primera persona, eso sí, pero algunos sí batallan para entender que hay que escribir en tercera persona. (Profesor \#3)

En las muestras de escritura, este fenómeno puede encontrarse en varios documentos:

Mi perspectiva respecto al uso de las tecnologías para la educación está basada en la experiencia. (Muestra \#4)

El segundo ejemplo que me parece adecuado es cómo ha surgido una corriente llamada los Boocktub. (Muestra \#9)

Por otro lado, todo el grupo docente entrevistado indica un correcto uso del vocabulario académico, técnico y científico que sus estudiantes manejan en sus escritos, sin embargo, sí encuentran fenómenos como la repetición recurrente de una palabra que podría evitarse a través del uso de deícticos, catafóricos y anafóricos, o la elisión del término.

Esto también puede identificarse en algunas de las muestras textuales de estudiantes:

En definitiva, no es algo moderno. El papel de la tecnología con fines educativos es una cuestión que lleva varios años. El uso de la tecnología en educación brinda orientación sobre las actuales herramientas tecnológicas y la gestión de la información. Así puedo expresar mi postura positiva ante el uso de la tecnología actual en la educación. (Muestra \#11)

Asimismo, cuatro de los cinco docentes aluden al uso limitado en conectores y marcadores textuales, y a la repetición de los pocos que usan:

Incluso cuando refieren a los autores, puede ser que siempre escriban "de acuerdo con" "de acuerdo con" ¿verdad? Entonces si pueda darse, ese es mucho más frecuente. (Profesor \#5)

En las muestras escritas del estudiantado se puede detallar también este asunto, pues el uso de conectores es escaso o nulo (Muestras \#3, Muestra \#5, Muestra \#8).

Otro de los aspectos en el identifican dificultades por parte de estudiantes es en el uso de palabras y expresiones más propios de la oralidad que del registro escrito:

Hay casos donde escriben como si estuvieran hablando. (Profesor \#2)

He notado que, en ocasiones, que pareciera como si, como si, es como si te estuvieran platicando. (Profesor \#4) 
http://doi.org/10.15359/ree.25-2.4

http://www.una.ac.cr/educare

educare@una.ac.cr

Tal aspecto también pudo evidenciarse en algunas de las muestras de texto de estudiantes:

La idea de hacer la investigación sobre evaluación surge del trabajo arduo de año tras año por mejorar este aspecto en la institución y aunque se hacen mejoras no se logra estar satisfecho con la evaluación en preescolar. (Muestra \#3)

Esto también coincide con lo reportado en estudios previos, en los que se argumenta que aspectos como el uso de la primera persona del singular, la ausencia de deícticos, catafóricos y anafóricos, expresiones de la oralidad, la falta de palabras conectores o marcadores textuales y las repeticiones resultan recurrentes en los escritos del estudiantado (Aguilar González y Fregoso Peralta, 2016; Rodríguez Hernández y García Valero, 2015).

\section{Oración y párrafo}

En cuanto a la construcción de oraciones y párrafos, el personal docente destaca la coherencia semántica, tanto para la construcción de ideas locales al interior del párrafo, como para la expresión de significados globales en el texto, lo que implica que el estudiantado tiene claridad sobre los conceptos o contenidos conceptuales que escribe y como los jerarquiza en el texto escrito. Sin embargo, señala que existen algunas dificultades en la construcción interna de párrafos por desequilibrio en la extensión de las oraciones, por la poca delimitación entre unas oraciones y por dificultades en signos de puntuación que no permiten establecer claramente el final de una oración y el inicio de otra:

En la construcción de los párrafos, sí se pueden ver algunas dificultades, puede ser que hagan párrafos muy grandes, de 15 o 20 renglones, y también se ve frecuentemente que usan mucho el punto y coma en lugar de la coma y se ve que usan muy poco el punto seguido. (Profesor \#4)

El profesorado también identifica deficiencias a la hora de articular unos párrafos con otros:

Hay otros alumnos que pueden batallar y se les señala que, bueno a lo mejor tal párrafo, o tal idea quedaría mejor en otro apartado ¿verdad? Para que lo revisen. (Profesor \#3)

Estas dificultades también son visibles en las muestras de textos, en las que se encuentran varios ejemplos de problemas en la construcción interna y desequilibrio entre la forma, la estructura y la extensión de los párrafos (Muestra \#3, Muestra \#7, Muestra \#9) y también dificultades en la articulación de distintos párrafos (Muestra \#3, Muestra \#5, Muestra \#11).

Las distintas características problemáticas en la construcción de párrafos halladas aquí han sido identificadas en otros estudios (Aguilar González y Fregoso Peralta, 2016; Arnoux et al., 2004; Pereira y di Stefano, 2007). 
http://doi.org/10.15359/ree.25-2.4

\section{Voz propia, citación y referenciación}

Si bien es cierto que el estudiantado debe partir de concepciones sobre los cuales se ha documentado, también es cierto que la escritura debe ser una construcción propia en la que su propia voz se articule con la de sus fuentes. Tanto la cita textual como el parafraseo son las formas más recurrentes. No obstante, el profesorado reconoce dificultades en ambas. En cuanto a las citas, señalan dos aspectos por mejorar: el exceso en el uso de citas y la poca articulación de la cita con su propio discurso.

Hay quienes sigueny basan sus textos en experiencias, luego alguna cita, que están relacionada y luego cierran, o más bien saturan de citas que no tienen mucho que ver. (Profesor \#2)

Pero más usual es el párrafo de cita, y luego otra cita y otra, y así. (Profesor \#4)

Este asunto también es evidenciable en algunas de las muestras, en las cuales se encuentran citas que abarcan incluso el párrafo entero, y cuya integración con el resto del discurso es poco clara (Muestra \#2, Muestra \#3, Muestra \#11).

En lo tocante al parafraseo, resaltan dificultades en la integración de las ideas, ya sea porque no hay una elaboración propia del conocimiento a partir de lo que leen, sino una transcripción de su fuente, lo que podría llevar incluso al plagio, o ya sea porque se les dificulta integrar el conocimiento de distintas fuentes para un mismo concepto.

Otra cosa en la que ellos batallan es la integración de las ideas con varios autores, y que al final del párrafo pongan a tres o cuatro autores, por orden alfabético. $O$ sea, esto lo dijeron estos cuatro, que integren, lo que dijeron en común tres autores o dos autores, también batallan para eso. (Profesor \#3)

El asunto del plagio se ha identificado por el profesorado en contadas ocasiones, generalmente al comienzo del proceso académico y no parte de una intención de hacer pasar el escrito ajeno como propio, sino como parte del desconocimiento de cómo hacer una cita o un parafraseo correcto, con la correspondiente referencia. Asimismo, señalan que una vez se ha trabajado con sus estudiantes el modo correcto de hacer la cita y la referencia, no se presentan casos de plagio.

Cuando son los aportes iniciales ocurre eso, que ponen ideas pegadas que, son citas textuales y no la declaran así, no hacen paráfrasis, pero andan pegando, pegando, por el desconocimiento mismo de cómo estructurar. (Profesor \#2).

La dificultad para articular la voz propia con la selección de información a partir de fuentes, se ha explicado como un aspecto recurrente en la escritura del estudiantado (Aguilar González y Fregoso Peralta, 2016; Arnoux et al., 2004; Rodríguez Hernández y García Valero, 2015). 
http://doi.org/10.15359/ree.25-2.4

http://www.una.ac.cr/educare

educare@una.ac.cr

Los hallazgos de este estudio pueden ser esquematizados como se presentan en la Figura 3. Allí puede observarse la forma en que la competencia comunicativa escrita del estudiantado, específicamente las dificultades en ella, se revelan en características de la escritura propiamente dicha, como la documentación previa a la escritura, los aspectos formales como ortografía gramática y léxico, los aspectos textuales como oración y párrafo y la articulación de la voz propia con referentes externos. También se halla que existen características contextuales que influyen en las dificultades de la competencia, como la formación profesional previa, las habilidades particulares y la experiencia en escritura académica, entre otros.

Figura 3: Hallazgos principales de este estudio

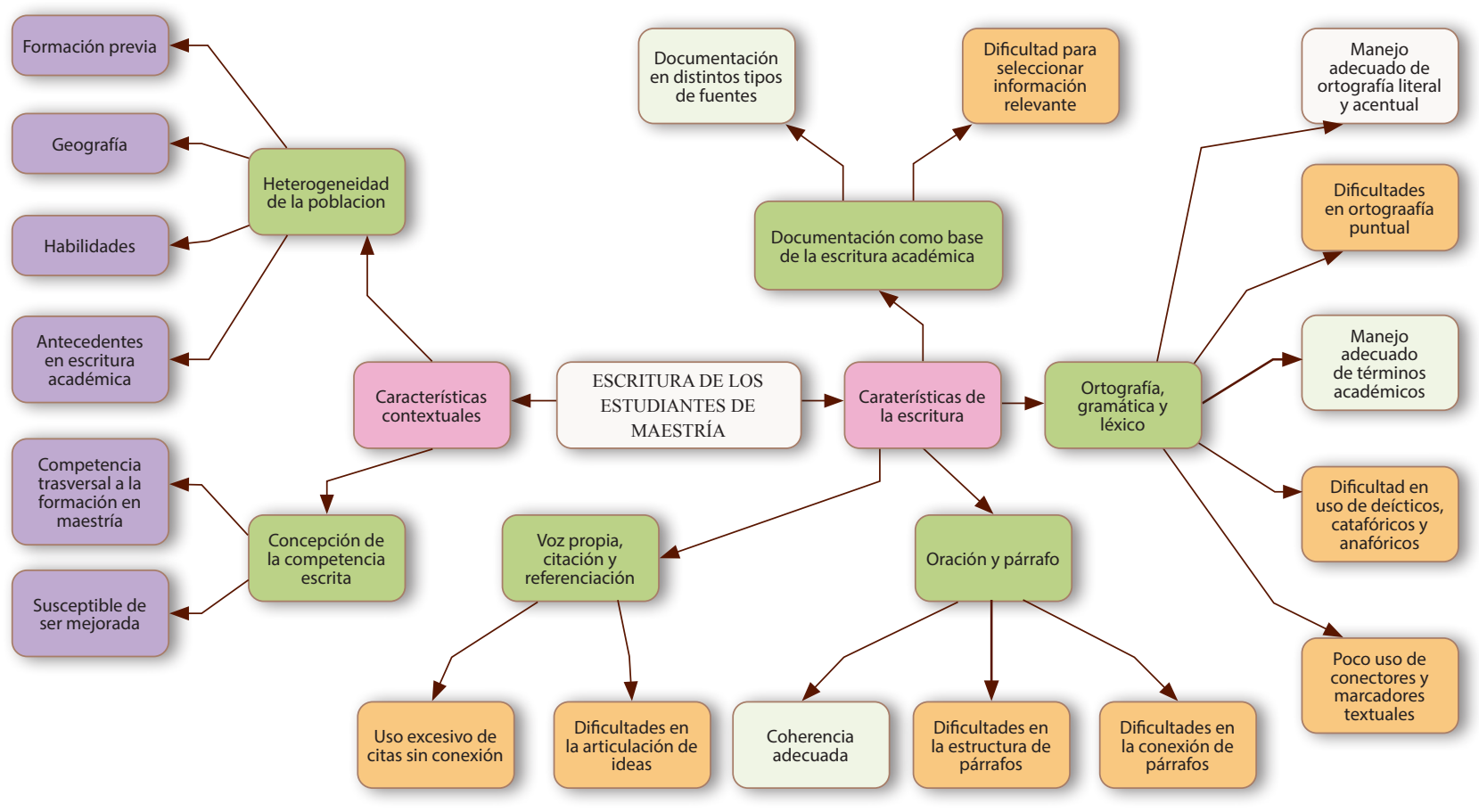

Nota: Elaboración propia.

\section{Discusión}

Las dificultades en la competencia comunicativa escrita de estudiantes de maestría, evidenciadas a través de sus escritos y referida por sus docentes, pueden ser identificables desde cuatro ángulos aquí presentados: 
http://doi.org/10.15359/ree.25-2.4

1. Documentación como base de la escritura: si bien es cierto que el estudiantado se documenta en distintos tipos de fuentes, se identifica una dificultad para seleccionar la información relevante y distinguirla de la que no lo es.

2. Ortografía, gramática y léxico: aunque existe un manejo adecuado de la ortografía acentual y literal, y del uso de vocabulario académico, existen dificultades en el uso de signos de puntuación, uso de deícticos catafóricos y anafóricos, uso de conectores y marcadores textuales.

3. Oración y párrafo: a pesar de que existe un adecuado manejo de la coherencia textual, se hallan dificultades en el uso de conexión entre párrafos, así como en la estructura de estos a lo largo del texto.

4. Voz propia, citación y referenciación: se encuentran dificultades en la articulación entre su propia voz con respecto a las fuentes documentales, además de un uso excesivo de citas sin conexión.

Algunas de estas dificultades han sido también identificadas en estudios previos (Aguilar González y Fregoso Peralta, 2016; Carlino, 2004; Rodríguez Hernández y García Valero, 2015; Salinas Padilla et al., 2009), lo cual puede interpretarse como un fenómeno generalizado en la escritura académica en los estudios de posgrado, que bien podría corresponder a las dinámicas propias del desarrollo de la competencia comunicativa escrita.

Por lo que respecta al contexto del estudiantado de este estudio, al igual que en otros estudios (Arnoux et al., 2004; Bandenhorst y Guerin, 2016; Martín Torres, 2012) se conceptúa la escritura como una competencia transversal a los procesos formativos propios en este nivel educativo. Esta competencia no se presenta como algo culminado o finalizado, incluso cuando el estudiantado cuenta con una formación profesional previa, sino que es una competencia que puede pasar por distintos estadios y que puede ser mejorada con la práctica y la capacitación. En este continuo mejoramiento, los recursos que utilizan sus docentes, como las sesiones, la retroalimentación, el uso de un manual (que en este caso particular es parte de la escuela o facultad), el uso de fichas bibliográficas y de otros recursos externos a la clase (una página web especializada y la biblioteca, ambas pertenecientes a la universidad) se convierte en herramientas intencionadas para el mejoramiento de esta competencia que, además, revela un compromiso por parte de docentes e institución por apoyar el proceso de sus estudiantes.

La heterogeneidad de la población en cuanto a su formación previa, motivaciones, procedencias y niveles de dominio de la escritura son aspectos que no solamente se observan en este estudio (Aguilar González y Fregoso Peralta, 2016; Salinas Padilla et al., 2009; StillmanWebb, 2016), sino que son recurrentes en los postgrados y requieren dinámicas de trabajo particulares para el fortalecimiento de esta competencia de acuerdo con cada caso. Asimismo, 
http://doi.org/10.15359/ree.25-2.4

http://www.una.ac.cr/educare

educare@una.ac.cr

este trabajo sostenido a través del tiempo refleja una progresión en el mejoramiento de la escritura, a la par de otras competencias propias que se desarrollan en este nivel formativo.

Dado que el presente estudio pretende constituirse en un diagnóstico para identificar aspectos a mejorar en la escritura académica del alumnado, es fundamental reconocer las características generales de la población y las concepciones que se tienen en torno a la escritura, así como las herramientas con las que cuenta en su contexto.

Con respecto a las características propias de la escritura del estudiantado, a partir de la concepción de docentes y las muestras de escritura analizadas, se permitieron determinar tanto fortalezas como áreas de oportunidad. Los aspectos mencionados en este trabajo como características susceptibles de mejora, también han sido evidenciados en otros estudios, lo que significa que más allá de ser rasgos propios derivados de un contexto institucional, son elementos recurrentes de la escritura académica en el nivel educativo del postgrado.

Es importante que un estudio diagnóstico identifique tanto las características poblacionales, así como las contextuales y las referidas propiamente a las áreas de oportunidad en la escritura del estudiantado. Por esto, en estudios futuros que aborden esta misma temática podrían tenerse en cuenta otras consideraciones. Si bien es cierto que este estudio exploró los tres aspectos, las limitaciones de tiempo no permitieron realizar una caracterización más profunda y más detallada del estudiantado, de todas las herramientas utilizadas en los cursos, y de las progresiones que se pueden dar en la escritura a través del tiempo. De igual manera, el presente estudio no contempló entrevistas a estudiantes, no obstante, en un estudio a futuro su perspectiva podría ser el paso inicial para hallar el camino hacia la solución de las dificultades halladas. Además, también sería deseable una muestra más grande de los documentos de estudiantes para identificar más fenómenos que pueden ser susceptibles de ser mejorados.

Aun así, los datos encontrados en este estudio permiten realizar una aproximación a las fortalezas y áreas de mejora en la escritura de estudiantes en este contexto, sobre los cuales se recomienda trabajar posteriormente para generar herramientas y recursos que permitan capacitar en las dificultades que se pudieron encontrar $y$, con ello, mejorar la competencia comunicativa escrita.

\section{Referencias}

Aguilar González, L. E. y Fregoso Peralta, G. (2016). El reto de la escritura académica en posgrado. Universidad de Guadalajara, Centro Universitario de Ciencias Sociales y Humanidades. http://repositorio.cualtos.udg.mx:8080/jspui/handle/123456789/605

Argudín, Y. y Luna, M. (2005). Aprender a pensar escribiendo bien. Desarrollo de habilidades para escribir. Trillas. 
http://doi.org/10.15359/ree.25-2.4

Arnoux, E., Borsinger, A., Carlino, P., Di Stefano, M., Pereira, C. y Silvestre, A. (2004). La intervención pedagógica en el proceso de escritura de tesis de posgrado. Revista de La Maestría en Salud Pública, 2(3), 1-16. https://www.aacademica.org/paula.carlino/169

Bandenhorst, C. y Guerin, C. (2016). Post/graduate research literacies and writing pedagogies. En C. Bandenhorst \& C. Guerin (Eds.), Research Literacies and Writing Pedagogies for Masters and Doctoral Writers (pp. 3-28). Brill. https://doi.org/10.1163/9789004304338

Bosio, I.V. (2018). ¿Podemos mejorar la calidad de la escritura en el posgrado? Algunas respuestas a partir de un proceso de investigación-acción. Revista Brasileira de Linguística Aplicada, 18(4), 737-769. https://doi.org/10.1590/1984-6398201812959

Carlino, P. (2003). Alfabetización académica: Un cambio necesario, algunas alternativas posibles. Revista Educere: Investigación Arbitrada, 6(20), 409-420. https://www.aacademica.org/ paula.carlino/23

Carlino, P. (2004). El proceso de escritura académica: Cuatro dificultades de la enseñanza universitaria. Educere, Revista Venezolana de Educación, 8(26), 321-327. http://www.redalyc. org/articulo.oa?id=35602605

Carlino, P. (2005). Escribir, leer y aprender en la universidad. Una introducción a la alfabetización académica. Fondo de Cultura Económica.

Carlino, P. (2013). Alfabetización académica Diez años después. Revista Mexicana de Investigación Educativa, 18(57), 355-381. http://www.scielo.org.mx/scielo.php?script=sci arttext\&pid $=\mathrm{S} 1405-66662013000200003$

Cassany, D. (2004). Construir la escritura. Paidos.

Cassany, D. (2006). Taller de textos. Leer, escribir y comentar en el aula. Paidos.

Denzin, N. K. y Lincoln, Y. S. (2011). Introduction: The discipline and practice of qualitative research. En N. K. Denzin e Y. S. Lincoln (Eds.), The sage handbook of qualitative research (pp. 1-19). SAGE.

Flores Aguilar, M. D. (2014). La competencia comunicativa escrita de los estudiantes de ingeniería y la responsabilidad institucional. Innovación Educativa, 14(65), 43-60. http://biblat.unam. $\mathrm{mx} / \mathrm{es} / \mathrm{revista} / \mathrm{innovacion}$-educativa/articulo/la-competencia-comunicativa-escrita-delos-estudiantes-de-ingenieria-y-la-responsabilidad-institucional

García Gutiérrez, A. (2002). Tratamiento y análisis de la documentación. En D. Vizcaya Alonso (Ed.), Selección de lecturas: Fundamentos de la organización de la información (pp. 72-94). Empresa Editorial Pligráfica Félix Varela. 
http://doi.org/10.15359/ree.25-2.4

http://www.una.ac.cr/educare

educare@una.ac.cr

Hayes, J. R. (1996). A new framework for understanding cognition and affect in writing. En C. M. Levy y S. Ransdell (Eds.), The science of writing: Theories, methods, individual differences, and applications (pp. 1-27). Lawrence Erlbaum.

Martín Torres, G. G. (2012). La escritura de tesis de posgrado en el área de investigación educativa. El acompañamiento, una pieza clave. Revista de Investigación Educativa, 15, 69-86. https:// doi.org/10.25009/cpue.v0i15.21

Maxwell, J. A. (2013). Qualitative research design. An interactive approach (3.ed.). SAGE.

Padilla, C. (2016). Desafíos epistémicos y argumentativos en la escritura de postgrado: Géneros científico-académicos y trayectorias de maestrandos y doctorandos. Traslaciones. Revista Latinoamericana de Lectura y Escritura, 3(6), 165-196. http://revistas.uncu.edu.ar/ojs/index. php/traslaciones/article/view/813

Pereira, C. y di Stefano, M. (2007). El taller de escritura en posgrado: Representaciones sociales e interacción entre pares. Revista Signos, 40(64), 405-430. https://doi.org/10.4067/s0718$\underline{09342007000200007}$

Radloff, A. y de la Harpe, B. (2000). Helping students develop their writing skills - A resource for lecturers. En L. Richardson y J. Lidstone (Eds.), Flexible Learning for a Flexible learning for a flexible society (pp. 566-573). Actas de la Conferencia Higher education research and development in south Asia Conference. Curtin University of Technology. https://www. ascilite.org/conferences/aset-archives/confs/aset-herdsa2000/procs/radloff-a.html

Rodríguez Hernández, B. A. y García Valero, L. B. (2015). Escritura de textos académicos: Dificultades experimentadas por escritores noveles y sugerencias de apoyo. Revista de Investigación Educativa, 20, 249-265. https://doi.org/10.25009/cpue.v0i20.1332

Ruiz Pérez, R. (1992). El análisis documental: Bases terminológicas, conceptualización y estructura operativa. Editorial Universidad de Granada.

Sacks, H., Schegloff, E. A. y Jefferson, G. (1974). A simplest systematics for the organization of turn-taking for conversation. Linguistic Society of America, 50(4), 696-735. https://doi. org/10.1353/lan.1974.0010

Salinas Padilla, H. A., Irizar Past, G. D. y López Jiménez, A. G. (2009). Impacto de los cursos de taller de lectura y redacción en los estudiantes de la DES-DAEH. En X Congreso Nacional de Investigación Educativa (pp. 1-17). Consejo Mexicano de Investigación Educativa. http://www.comie.org.mx/congreso/memoriaelectronica/v10/pdf/area tematica 05/ ponencias/0732-F.pdf 
http://doi.org/10.15359/ree.25-2.4

http://www.una.ac.cr/educare educare@una.ac.cr

Stillman-Webb, N. (2016). Writing beliefs and mentoring practices: Advisor perspectives on post/graduate writing instruction in the sciences. En C. Bandenhorst y C. Guerin (Eds.), Research Literacies and Writing Pedagogies for Masters and Doctoral Writers (pp. 257-276). Brill. https://doi.org/10.1163/9789004304338 015

Valenzuela González, J. R. y Flores Fahara, M. (2012). Fundamentos de investigación educativa (Vol. 2). Editorial Digital del Tecnológico de Monterrey.

Vela, F. (2001). Un acto metodológico básico de la investigación social: La entrevista cualitativa. En M. L. Tarrés (Coord.), Observar, escuchar y comprender: Sobre la tradición cualitativa en la investigación social (2. ${ }^{a}$ ed., pp. 63-95). FLACSO. 